\title{
Depairing Current at High Magnetic Fields in Vortex-Free High Temperature Superconducting Nanowires
}

Victor Rouco, ${ }^{\dagger}, \|$ Carles Navau, ${ }^{\ddagger}$ Nuria Del-Valle, ${ }^{\ddagger}$ Davide Massarotti, $₫$ Gian Paolo

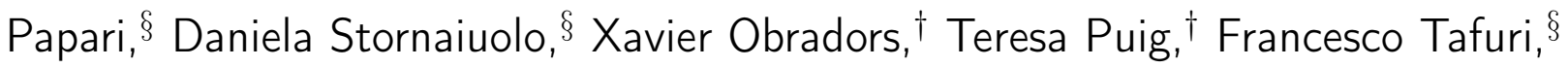
Alvaro Sanchez, ${ }^{\ddagger}$ and Anna Palau* ${ }^{* \dagger}$

$\dagger$ Insitut de Ciencia de Materials de Barcelona, CSIC, Campus de la UAB, 08193 Bellaterra, Catalonia, Spain

$\ddagger$ Departament de Fisica, Universitat Autonoma de Barcelona, 08193 Bellaterra, Barcelona, Catalonia, Spain

IDipartimento di Ingegneria Elettrica e delle Tecnologie dell'Informazione, Università degli Studi di Napoli Federico II, 80125 Napoli, Italy

$\S$ Dipartimento di Fisica, Universita degli Studi di Napoli Federico II, 80126 Napoli, Italy ||Current address: Univ Paris Sud, CNRS, Unite Mixte Phys, Thales, France

E-mail: palau@icmab.es

\begin{abstract}
Superconductors are essential in many present and future technologies, from largescale devices for medical imaging, accelerators, or fusion experiments to ultralow-power superconducting electronics. ${ }^{1}$ However, their potential applicability, and particularly that of high-temperature superconductors (HTS), is severely affected by limited performances at large magnetic fields and high temperatures, where their use is most needed.
\end{abstract}


One of the main reasons for these limitations are the presence of quantized vortices, whose movements result in losses, internal noise, and reduced performances. ${ }^{2,3}$ The conventional strategy to overcome flow of vortices is to pin them along artificial defects. ${ }^{3-7}$ Here we theoretically and experimentally demonstrate that critical-current density in high-temperature superconductors can reach unprecedented high values at high fields and temperatures, by preventing vortex entry. By tailoring the geometry, i.e. reducing the width, $W$, of nanowire-patterned HTS films, the range of the Meissner state, for which no vortices are present, is extended up to very large applied field values, on the order of $\sim 1 \mathrm{~T}$. Current densities of the order of the depairing current can be sustained under high fields, for a wide range of temperatures. Results may be relevant both for devising new conductors carrying depairing-current values at high temperatures and large magnetic fields and for reducing flux noise in sensors and quantum systems.

The discovery of high-temperature superconductors hold great promise to revolutionize the applications of superconducting technologies, strongly reducing system operating costs. Still, the potential of HTS materials is far from being fully achieved. The fundamental limits of HTS performance are delimited by the depairing critical-current density, $J_{c, d p}$, upper critical magnetic field, $\mu_{0} H_{c 2}$, and critical temperature, $T_{c}$. In particular for $\mathrm{YBa}_{2} \mathrm{Cu}_{3} \mathrm{O}_{7-\delta}(\mathrm{YBCO})$ cuprate superconductors, with characteristic coherence length, $\xi_{0}=\xi(T=0 \mathrm{~K}) \sim 1.5-2 \mathrm{~nm}$, and penetration depth, $\lambda_{0}=\lambda(0 \mathrm{~K}) \sim 150-200 \mathrm{~nm}$, one obtains a Ginzburg-Landau depairing current, $J_{c, d p}(0 \mathrm{~K}) \sim 100-300 \mathrm{MA} / \mathrm{cm}^{2}, \mu_{0} H_{c 2}(0 \mathrm{~K})>100 \mathrm{~T}$, and $T_{c} \sim 92 \mathrm{~K} .{ }^{2,31}$ However, energy dissipation in the mixed state, in which vortices penetrate the superconductor, severely limits their superconducting performance, thus becoming a critical issue in most practical applications. Motion of vortices under the Lorentz force determines the value of $J_{c}$, which is specially reduced at high temperatures, where thermal effects become very relevant, and high applied fields, which are the situations where HTS could offer more advantages. ${ }^{2}$ An additional important fact is that most common HTS materials are thin strips (e. g. coated conductors), with very large demagnetizing fields. As a result, in practice any value however small of applied magnetic field perpendicular to the plane of 
the superconductor, results in vortex penetration. Enhancing current-density values in the mixed state has been a long-standing challenge. To reduce the $J_{c}$ dependence on magnetic field, artificial vortex pinning sites are added to the superconductors, requiring a difficult optimization of the pinning landscape for the specific operating conditions (temperature, and magnetic field value and orientation). ${ }^{5-7}$ Moreover, vortex motion leads to flux noise that limits the operation of sensitive superconducting electronics ${ }^{9}$ and quantum applications. ${ }^{10}$ Therefore, achieving large currents in the superconductor in a vortex-free regime becomes crucial for cutting-edge applications for both large and small scales.

Reduction of the dimensionality in oxide functional materials has received growing scientific and technological interest due to the range of new properties and obtained funcionalites, as compared with the bulk. ${ }^{12,13,15}$ Thanks to advances in high-resolution lithography techniques, it is now possible to fabricate complex nanostructures in epitaxial functional oxides, without damaging their physical properties. ${ }^{16}$ When a superconducting film is shrunk to mesoscopic length scales, both the supercurrent distribution and the properties of vortex matter are strongly influenced by the sample topology and size. ${ }^{17-19}$ In particular, there are two effects that may be specially relevant for technological applications.

On the one hand, the critical current density may be enhanced up to the depairing limit, by reducing the cross section to the characteristic lengths $\xi$ and $\lambda$. This effect has been theoretically predicted in several studies? ? and experimentally observed in both conventional type II superconductors and high temperature superconductors. Some examples in conventional superconductors include $\mathrm{Al},{ }^{?} \mathrm{Nb}$ or $\mathrm{Mo}_{0.7} \mathrm{Ge}_{0.3} ?$ thin films, with thickness $t \sim 20-50$ $\mathrm{nm}$, patterned with micrometric wires, $W \sim 1-2 \mu \mathrm{m}$. Moreover, robust values of self-field $J_{c, d p}$, over the full superconducting temperature range, could also be achieved in complex structures consisting of two-dimensional Nb thin films $(t=11 \mathrm{~nm})$ patterned with nanomeshes (34nm spatial periodicity). Although being a more challenging task, theoretical depairing currents at zero applied field can also be observed in high temperature superconductors. For example in YBCO nanowires with cross sections of $50 \times 50 \mathrm{~nm}^{2}$, ascribed to current crowding 
effects $^{22 ?}$ or $(\mathrm{Ba}, \mathrm{K}) \mathrm{Fe}_{2} \mathrm{As}_{2}$ microbridges $(t=2 \mu \mathrm{m})$ with nanoscale thickness $(t \sim 90 \mathrm{~nm})$.

On the other hand, geometry constrains are known to be responsible for vortex exclusion and rearrangement processes. It is theoretically predicted that vortices are completely expelled from a thin superconducting strip, of width $W$ larger than the in-plane penetration depth, below a critical field $B_{0} \sim 1 / W^{2}$, that only depends on the width of strip with a pre-factor that slightly depend on the model considered. ${ }^{10,11,26}$ This effect have been experimentally proved in micrometric $\mathrm{Nb}$ and $\mathrm{YBCO}$ thin strips through vortex imaging techniques. In the first case, by reducing the width of the strip down to $W=1.6 \mu \mathrm{m}$ first vortex penetration was observed at $\sim 2 \mathrm{mT},{ }^{10}$ whereas in the later vortex expulsion until $\sim$ $0.1 \mathrm{mT}$ was obtained in strips of $t=6 \mu \mathrm{m} .{ }^{26}$ In both cases, vortex arrangement in single row at the centre of the strip was observed, when the applied field reached $B_{0}$. These experimental al theoretical works anticipated the potential of nano-metric structures to eliminate noise linked to vortex motion up to high magnetic fields. First vortex penetration at fields of $\sim 1 \mathrm{~T}$ was experimentally demonstrated on $\mathrm{W}$-based nanowires of $t=30 \mathrm{~nm}$ and $W=$ $50 \mathrm{~nm},{ }^{24}$ by means of magnetoresistance measurements. In the same work recovery of the superconducting state at even large fields was reported due to vortex self-arresting processes, opining the path for the design of loss-less superconducting devices at high fields and temperatures. Magnetoresistance measurements were also used to study geometrical effects on the vortex lattice in high temperature superconducting nanowires. ${ }^{23}$ All this studies were performed close to the irreversibility line by using low applied currents.

However, up to now, experimental evidence of the two effects - i.e., vortex-free state in a wide range of the $H$ - $T$ phase diagram, in nanowires sustaining large depairing critical current densities, has not been achieved yet. In this work provide experimental evidence that, by combining this two well known geometrical effects, in technologically relevant $\mathrm{YBCO}$ nanowires with properly adjusted dimensions, key $J_{c}(H)$ performances may be achieved. Nanowires of widths $W \sim 80-100 \mathrm{~nm}$, and thickness, $t=150 \mathrm{~nm}$, show a basically temperature-independent $J_{c}(H)$ plateau, with critical current density values approaching par- 
ticularly large YBCO depairing limit, up to $\mu_{0} H_{a} \sim 1 \mathrm{~T}$. Such values of current at high fields are above those ever been achieved using conventional flux-pinning strategies. It is worth noting that the obtained results are not limited to a thin film geometry offering enhanced flexibility in the device design. We first present the theoretical basis of how a tailored geometry at the nanoscale leads to vortex exclusion up to high fields, and then experimentally confirm the validity of the proposed strategy in actual superconducting YBCO nanowires.

We consider a superconducting slab extending in the $y$ direction, occupying the region $-W / 2<x<W / 2$, with thickness $t \gg W$ along the $z$ axis. A uniform external magnetic field $\mu_{0} H_{a}$ is applied along the $z$ direction. We use $\kappa=\lambda / \xi$, being $\lambda$ and $\xi$ the superconductor London penetration and coherence lengths, respectively. We assume $W \gg \xi$ and $\lambda \gg \xi$ $(\kappa \gg 1)$. In this geometry, for which demagnetizing effects are negligible $(t \gg W)$, it is energetically favourable to have vortices in the superconductor when the applied field reaches the first critical field $\mu_{0} H_{c 1}$, given by ${ }^{28}$

$$
\mu_{0} H_{c 1}=\frac{\Phi_{0}}{4 \pi \lambda^{2}}\left(\log \kappa+0.081-2 \sum_{n=1}^{\infty}(-1)^{n+1} K_{0}\left(\frac{W n}{\lambda}\right)\right)\left(1-\operatorname{sech}\left(\frac{W}{2 \lambda}\right)\right)^{-1}
$$

where $\Phi_{0}$ is the flux quantum, $\mu_{0}$ is the vacuum permeability, and $K_{i}()$ are the modified Bessel functions of the second kind and $i$-th order. The expression for $\mu_{0} H_{c 1}$ has the limits

$$
\begin{gathered}
\mu_{0} H_{c 1}(W \ll \lambda)=\frac{2 \Phi_{0}}{\pi W^{2}}\left[\log \left(1.78 \frac{W}{\pi \xi}\right)+0.081\right], \\
\mu_{0} H_{c 1}(W \gg \lambda)=\frac{\Phi_{0}}{4 \pi \lambda^{2}}\left(2 e^{-\frac{W}{2 \lambda}}+1\right)(\log \kappa+0.081) .
\end{gathered}
$$

For perfectly flat surfaces, surface barriers ${ }^{29}$ prevent vortices entering the slab up to a larger superheating field, $\mu_{0} H_{s}$, given by ${ }^{30}$

$$
\mu_{0} H_{s}=\frac{\Phi_{0}}{2 \pi \lambda^{2}} \cosh \left(\frac{W}{2 \lambda}\right) \operatorname{csch}\left(\frac{1}{\kappa}-\frac{W}{2 \lambda}\right) \sum_{n=0}^{\infty}\left[K_{1}\left(\frac{2 W n}{\lambda}+\frac{2}{\kappa}\right)-K_{1}\left(\frac{2 W(n+1)}{\lambda}-\frac{2}{\kappa}\right)\right]
$$

We plot in Fig. 1 as solid lines the dependence of $\mu_{0} H_{s}, \mu_{0} H_{c 1}$, and the limiting expres- 
sions upon the superconductor width $W$ (normalized to $\lambda_{0}$ ), given by the above analytic expressions, considering typical $\xi_{0}$ and $\lambda_{0}$ values for YBCO. Interestingly, Eq. (2) shows that for widths $W$ much smaller than $\lambda$ the field $\mu_{0} H_{c 1}$ for vortex penetration depends basically as $\Phi_{0} / W^{2}$, which can reach values on the order of $1 \mathrm{~T}$ for nanowires with sizes of tens of nanometers. Theory also indicates that $\mu_{0} H_{c 1}$ depends very little upon the temperature [only trough the factor $\xi$ inside the logarithm in Eq. (2)].

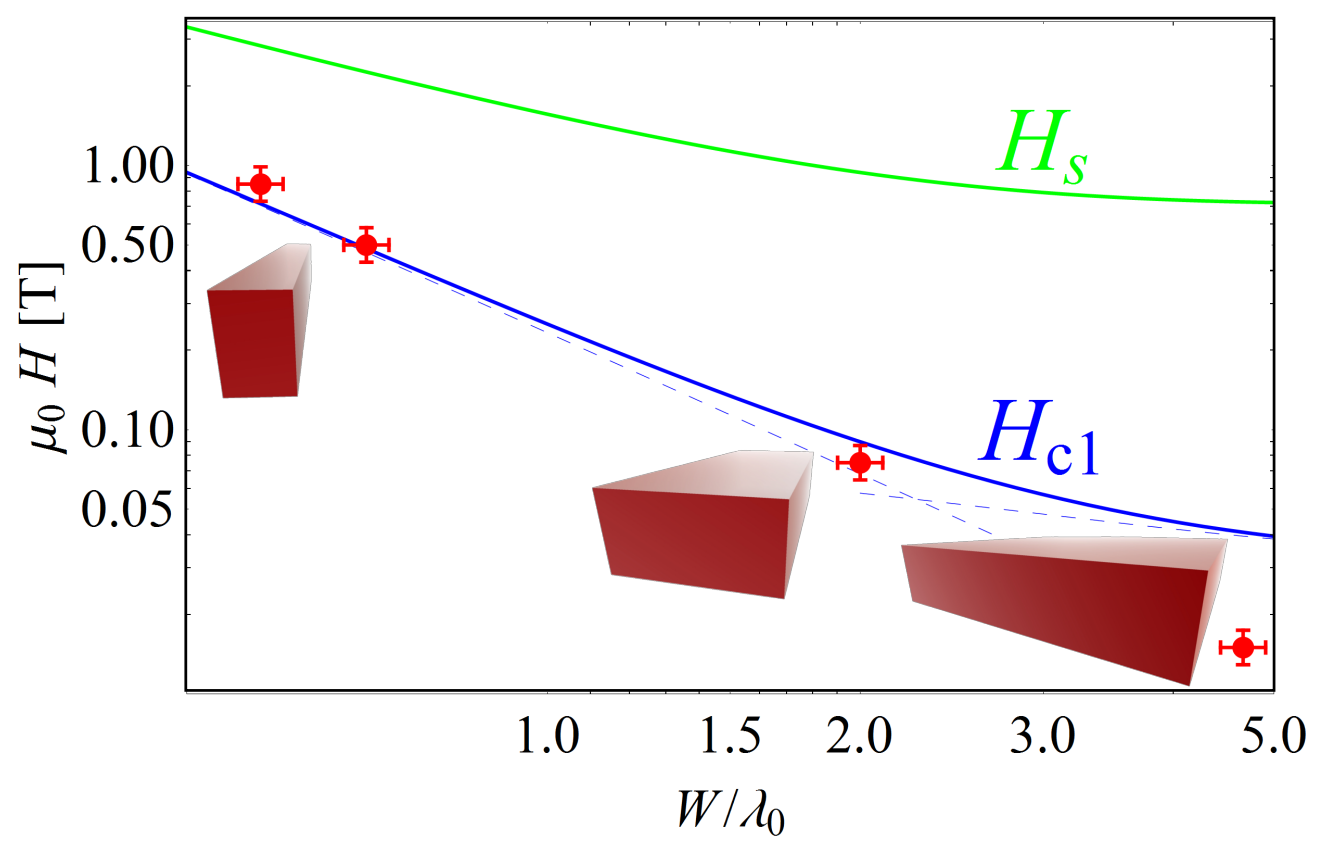

Figure 1: Magnetic fields for the entering of vortices: (blue) first penetration field $\mu_{0} H_{c 1}$ with the limiting expressions (dashed-blue); (green) supercooling field $\mu_{0} H_{s}$. Red dots corresponds to the experimental values of $\mu_{0} H_{\text {crit }}$. Figures depict the aspect ratio of different nanowires.

To experimentally confirm the theoretical predictions, we fabricate YBCO nanowires of different widths ranging from $W=80-700 \mathrm{~nm}$, with a fixed length of $1 \mu \mathrm{m}$ and thickness of 150nm (see Fig. 2a and Methods). All patterned nanowires had values of $T_{c} \sim 87-92 \mathrm{~K}$, indicating no significant degradation of the bulk superconducting properties. Figure 2b shows the self-field critical-current density, $J_{c, s f}$, at $5 \mathrm{~K}$, for several micro and nanowires of different widths. A systematic enhancement of $J_{c, s f}$ is observed when reducing $W$, reaching 
values that approach the Ginzburg-Landau depairing current, $J_{c, d p}$, in the limit $W<2 \lambda_{0}$. Although many of narrow nanowires show very high values of $J_{c, s f}(5 \mathrm{~K}) \sim 40-60 \mathrm{MA} / \mathrm{cm}^{2}$, the reproducibility of samples sustaining critical-current density values close to the depairing is low. At these small dimensions, sample inhomogeneities (porosity, nano-precipitates, or amorphisation during the patterning process) may damage some of the properties and reduce the effective nanowire cross section.

(a)

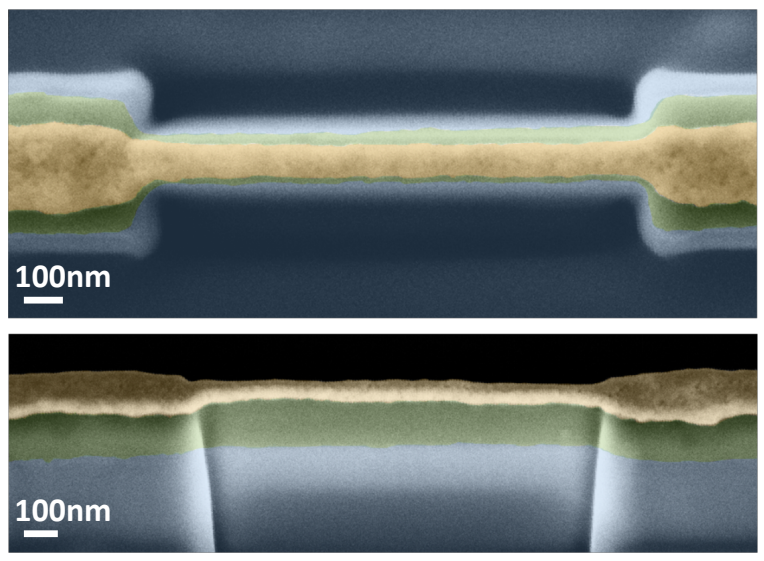

(b)

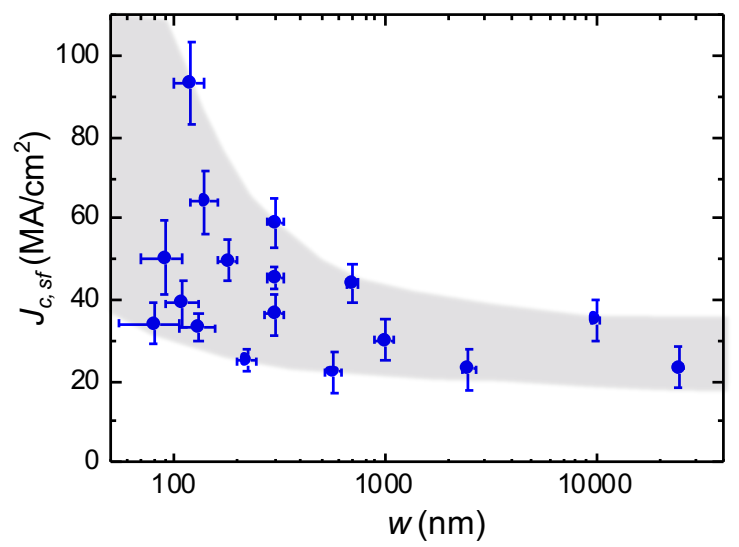

Figure 2: (a) False colour top-view (Top) and cross-section (Bottom) SEM image of a 80 nm-width, 150nm-thick nanowire. (b) $J_{c, s f}$ at $5 \mathrm{~K}$ for several YBCO micro and nanowires of different width.

The magnetic field dependence of $J_{c} / J_{c, s f}$ at $65 \mathrm{~K}$ for nanowires of different widths is presented in Fig. 3a. Results for a wider microwire of $W=30 \mu \mathrm{m}$ are added for comparison. For all nanowires $J_{c}$ is constant up to a critical magnetic field value, $\mu_{0} H_{c r i t}$. There is a systematic enhancement of $\mu_{0} H_{\text {crit }}$ with reducing $W$, with a plateau extended up to $\mu_{0} H_{\text {crit }} \sim 1 \mathrm{~T}$ for nanowires with $W=80-100 \mathrm{~nm}$. In macroscopic systems, the $J_{c}$ plateau is associated to a single-vortex pinning regime dominated by vortex-defect interactions, crossing over to a collective regime above $\mu_{0} H_{\text {crit }}$ when vortex-vortex interactions become important. ${ }^{32,33}$ In those samples, $\mu_{0} H_{\text {crit }}$ decreases with increasing the temperature due to thermally activated processes. ${ }^{34}$ However, in our nanowires the plateau can unambiguously be associated to the absence of vortices in the superconductor. A first evidence can be seen in Fig. 1 where we 
(a)

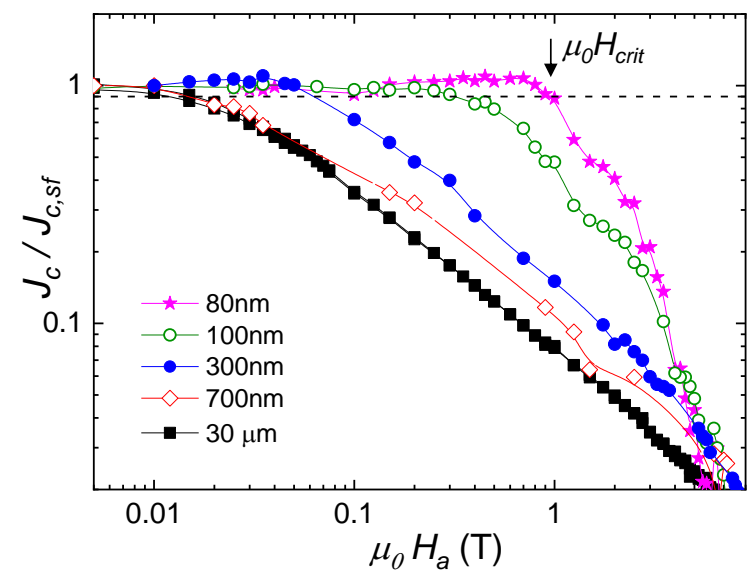

(b)

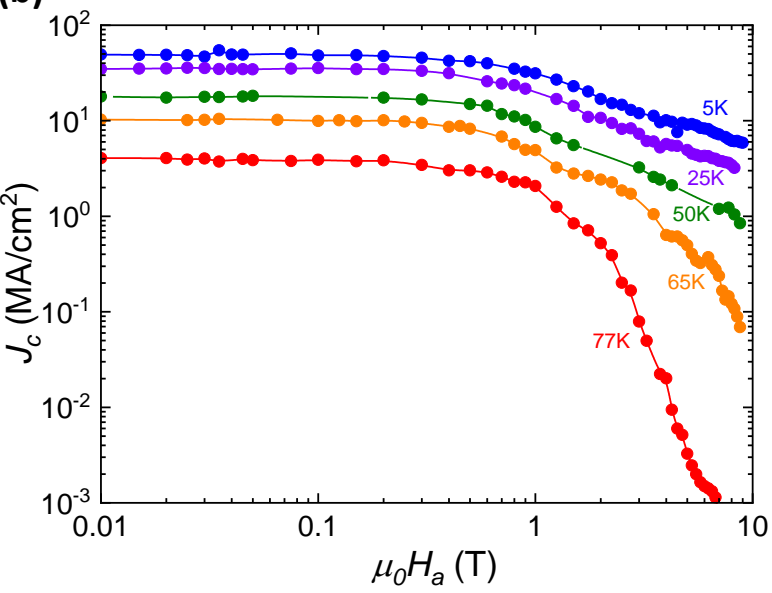

Figure 3: (a) Normalized critical-current density as a function of the applied magnetic field for nanowires of different widths and a microwire at 65K. Dashed line shows the criteria used to determine $\mu_{0} H_{\text {crit }}$ (depicted with an arrow for the nanowire of 80nm). (b) Applied-field dependence of $J_{c}\left(H_{a}\right)$ obtained for a nanowire of $W=100 \mathrm{~nm}$ at different temperatures.

plot the experimental values of $\mu_{0} H_{\text {crit }}$ for the nanowires with different widths $W$ together with the theoretical prediction for the field of vortex penetration. The experimental values match very well the theoretical $\mu_{0} H_{c 1}$ dependence; remarkably, this is a zero-parameter fit, since theoretical values only depend upon experimentally determined quantities. Only the experimental point at $W / \lambda_{0} \sim 5(W=700 \mathrm{~nm})$ departs from the expected line because in this case the original assumption of $t \gg W$ does not hold. Theory also predicts that if the smoothness of the nanowires surfaces is eventually improved, one could raise the field of penetration further up to values close to the superheating field values, $\mu_{0} H_{s}$ in Fig. 1 .

A further confirmation that vortices are absent in our nanowires comes from confirming the temperature independence of $\mu_{0} H_{\text {crit }}$ predicted by the theory. In Fig. 3b it is seen that $\mu_{0} H_{\text {crit }}$ for a nanowire with $W=100 \mathrm{~nm}$ remains basically constant in a large temperature window from $5 \mathrm{~K}$ to $77 \mathrm{~K}$. The temperature-independence of $\mu_{0} H_{c r i t}$ is also evidenced in Fig. 4a, where $\mu_{0} H_{\text {crit }}(T)$ obtained for nanowires of different widths and a microwire with $W=$ $30 \mu \mathrm{m}$ is plotted as a function of temperature. $\mu_{0} H_{\text {crit }}$ remains essentially constant throughout all temperature range for all nanowires, whereas for the microwire $\mu_{0} H_{\text {crit }}$ monotonically drops with the temperature, as expected considering thermally activated vortex motion. ${ }^{32,33}$ 
This confirms that vortices do not penetrate in our superconducting nanowires until the applied field reaches the basically temperature-independent $\mu_{0} H_{\text {crit }}$ [Eq. (2)].

(a)

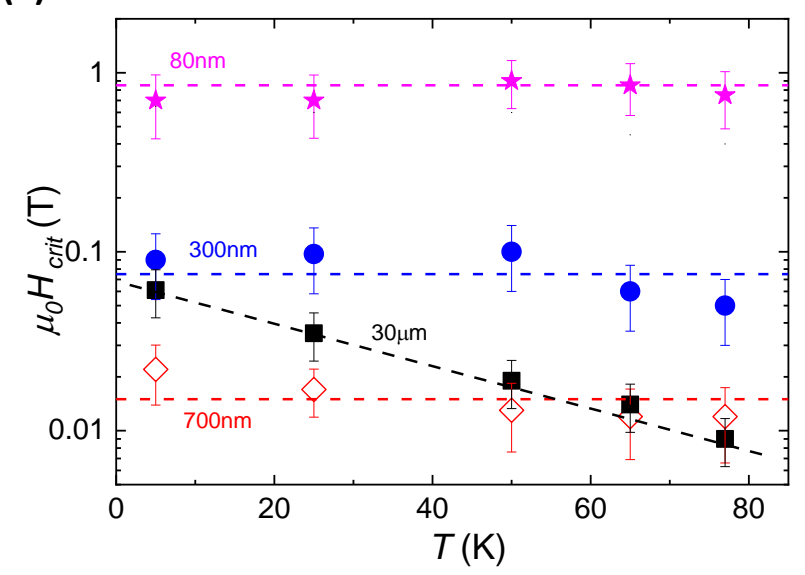

(b)

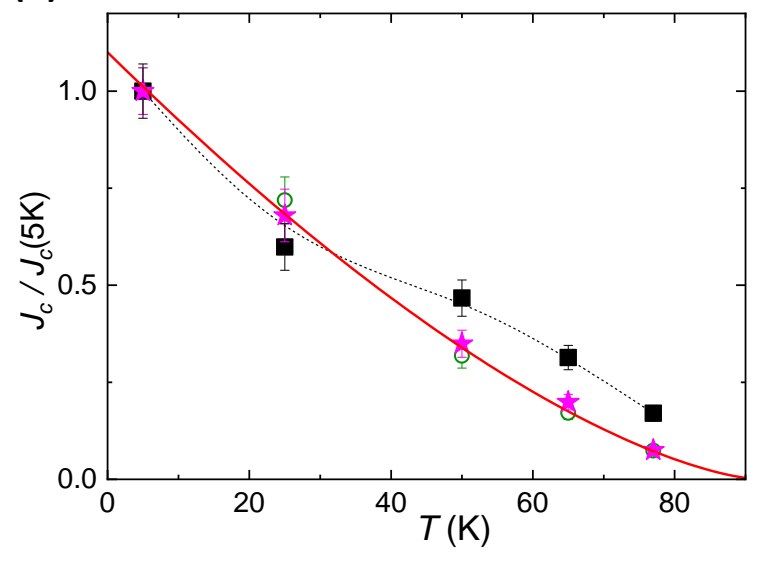

Figure 4: (a) Temperature dependence of $\mu_{0} H_{\text {crit }}$ for different nanowires and a microwire. Dashed lines are guide for the eye illustrating the $\mu_{0} H_{\text {crit }}(T)$ dependence. (b) Temperature dependence of self-field current density $J_{c, s f}$ normalized to its value at $5 \mathrm{~K}$, for two nanowires with $W=80 \mathrm{~nm}$ (pink stars) and 100nm (green open circles), and a microwire with $W=30 \mu \mathrm{m}$ (black squares). Solid red line shows the temperature dependence of the depairing current density, $J_{c, d p}$. Dashed black line is a guide for the eye

Figure $4 \mathrm{~b}$ shows the self-field $J_{c}$, normalized to its value at $5 \mathrm{~K}$, for two nanowires with $W=80 \mathrm{~nm}$ and $100 \mathrm{~nm}$ and, for comparison, a microwire with $W=30 \mu \mathrm{m}$. The experimentally observed temperature dependence of both nanowires can be well fitted with the Ginzburg-Landau depairing current temperature dependence, $J_{c, d p} \sim 1 /\left(\lambda^{2}(T) \xi(T)\right)$, providing evidence that critical-current density is achieving the theoretical depairing values. In contrast, the $J_{c}$ temperature dependence for the microwire, governed by vortex pinning, displays a different pattern.

Our results may have important consequences in practical applications. On the one hand, the fact that no vortices are present in our nanowires makes them excellent candidates to use them in noise-sensitive devices such as SQUIDs, superconducting qubits or sensors. ${ }^{9,10}$ On the other hand, the very large values of current density achieved at high applied fields and temperatures may be also relevant at large scale, with potential to improve the performance of actual thin strips (e. g. coated conductors). Large currents could eventually obtained by 
densely packing such nanowires, which may have large thickness, with barely any magnetic interaction among them. In conclusion, we have provided a novel avenue towards achieving unprecedentedly high values of currents at high field and temperatures in superconductivity applications, by tailoring the geometry to prevent vortex penetration rather than the conventional strategy of pinning vortices by defects.

\section{Methods}

Superconducting nanowires were fabricated by a top-down approach. The initial system consists of a c-axis oriented $\mathrm{YBa}_{2} \mathrm{Cu}_{3} \mathrm{O}_{7-\delta}$ 150nm-thick film grown on $\mathrm{LaAlO}_{3}$ substrate via Chemical Solution Deposition methodology. ${ }^{35}$ Silver contact pads were sputtered on the thin films and annealed under oxygen atmosphere at $450^{\circ} \mathrm{C}$ for 1 hour to ensure good electrical contacts with resistances below $10 \mu \Omega$. Photolithography and chemical etching were used to define microwires of width $\mathrm{W}=25 \mu \mathrm{m}$ and length $\mathrm{L}=100 \mu \mathrm{m}$, allowing current-voltage, I-V, characteristic measurements with the standard four-point method. Patterned microwires were protected with a 50nm gold layer grown by sputtering. The width of the microwires was finally reduced to the nanoscale by using Focused Ion Beam (FIB) [Zeiss 1560XB Cross Beam] lithography, following the process described in,${ }^{36}$ enabling to obtain nanowires with good superconducting performances. In field transport measurements were conducted in a commercial 9T Physical Property Measurement System (PPMS). The magnetic field was always applied parallel to the c-axis of the superconductor in a maximum Lorentz Force configuration. Critical temperature, $T_{c}$, was measured by recording the voltage under $10 \mu \mathrm{A}$ current. Critical-current density, $J_{c}$, was obtained by means of current bias transport measurements and using a $200 \mathrm{nV}$-criteria. The critical magnetic field value $\mu_{0} H_{\text {crit }}$ discussed below was determined at $0.9 J_{c, s f}$.

The theoretical formulas for the penetration fields in a type-II superconducting slab used in the text have been derived following analogous procedures as those by Abrikosov ${ }^{28}$ and 
Shmidt,${ }^{30}$ which dealt with the case of the slab in an applied magnetic field. In our case of transport currents, since the experimental values of currents have been on the order of $\sim 10 \mathrm{~mA}$, they generate a self-field of $\sim 2 \mathrm{mT}$, much less than the involved $\mu_{0} H_{c 1}$ or $\mu_{0} H_{s}$ values. For this reason, the field relevant for studying penetration has been considered to be the applied field and not the self-field of the transport currents.

\section{Acknowledgements}

We acknowledge financial support from Spanish Ministry of Economy and Competitiveness through the "Severo Ochoa" Programme for Centres of Excellence (SEV-2015-0496), COACHSUPENERGY project (MAT2014-51778-C2-1-R, co-financed by the European Regional Development Fund), and project MAT2016-79426-P (Agencia Estatal de Investigación

/ Fondo Europeo de Desarrollo Regional). We also thank support from the European Union for NANOCOHYBRI project (Cost Action CA 16218) and from the Catalan Government projects SGR-2017-1519 and 2017-SGR-105. A. S. acknowledges a grant from ICREA Academia, funded by the Generalitat de Catalunya. We acknowledge the use of ICTS-CNM facilities and ICMAB scientific and technical services. We thank Alexey V. Pan for fruitful discussions.

\section{References}

(1) S. Nishijima, S. Eckroad, A. Marian, K. Choi, W. S. Kim, M. Terai, Z. Deng, J. Zheng, J. Wang, K. Umemoto, J. Du, P. Febvre, S. Keenan, O. Mukhanov, L.D. Cooley, C. P. Foley, W. V. Hassenzahl, and M. Ixumi, Superconductivity and the environment: a Roadmap, Supercond. Sci. Technol. 26, 113001 (2013).

(2) D. Larbalestier, A. Gurevich, D. M. Feldmann, and A. Polyanskii, Superconducting materials for electric power applications. Nature 414, 368 (2001). 
(3) R. Wordenweber, Engineering of superconductors and superconducting devices using artificial pinning sites, Physical Sciences Reviews, De Gruyter, Chap. 2, 20178000 (2017).

(4) A. Palau, V. Rouco, R. F. Luccas, X. Obradors, T. Puig, Vortex dynamics in nanofabricated chemical solution deposition high-temperature superconducting films, Superconductors at the Nanoscale, De Gruyter, Chap. 6, 195 (2017).

(5) X. Obradors, T. Puig, Coated conductors for power applications: materials challenges. Supercond. Sci. Technol. 27, 044003 (2014).

(6) W. K. Kwok, U. Welp, A. Glatz, A. E. Koshelev, K. J. Kihlstrom, and G. W. Crabtree, Vortices in high-performance high-temperature superconductors. Reports Prog. Phys. 79, 116501 (2016).

(7) J. P. F. Feighan, A. Kursumovic, and J. L. MacManus-Driscoll, Materials design for artificial pinning centres in superconductor PLD coated conductors. Supercond. Sci. Technol. 30, 123001 (2017).

(8) M. Tinkham. Introduction to Superconductivity (New York: MacGraw-Hill) 103, 125 ( 1996).

(9) S. Savel'ev and F. Nori, Experimentally realizable devices for controlling the motion of magnetic flux quanta in anisotropic superconductors. Nat. Mater. 1, 179 (2002).

(10) J. M. Martinis, Superconducting phase qubits, Quantum Inf. Process 8, 81 (2009).

(11) K. Likharev, Sov. Radiophys. 14, 772 (1972).

(12) C. H. Ahn, Ferroelectricity at the Nanoscale: Local Polarization in Oxide Thin Films and Heterostructures. Science 303, 488 (2004).

(13) H. Y. Y. Hwang, Y. Iwasa, M. Kawasaki, B. Keimer, N. Nagaosa, and Y. Tokura, Emergent phenomena at oxide interfaces. Nat. Mater. 11, 103 (2012). 
(14) A. Palau, V. Rouco, R. F. Luccas, X. Obradors, and T. Puig, Vortex dynamics in nanofabricated chemical solution deposition high-temperature superconducting films. in "Superconductors at the Nanoscale From Basic Research to Applications", De Gruyter (2017).

(15) A. Kumar, "Functional Nanomaterials: From Basic Science to Emerging Applications," in Functional nanomaterials and their applications 201, 1 (2013).

(16) Zhang, Epitaxial patterning of thin-films: conventional lithographies and beyond. J. Micromechanics Microengineerign. Top. Rev 24, 093001 (2014).

(17) A. Bezryadin, Quantum suppression of superconductivity in nanowires. J. Phys. Condens. Matter 20, 043202, (2008)

(18) K. Xu and J. R. Heath, Long, Highly-Ordered High-Temperature Superconductor Nanowire Arrays, Nano Lett. 8, 3845 (2008)

(19) E. A. Dauler, M. E. Grein, A. J. Kerman, F. Marsili, S. Miki, S. W. Nam, M. D. Shaw, H. Terai, V. B. Verma, and T. Yamashita, Review of superconducting nanowire singlephoton detector system design options and demonstrated performance. Opt. Eng. 53, $081907(2014)$

(20) K. Xu, P. Cao, and J. R. Heath, Achieving the theoretical depairing current limit in superconducting nanomesh films. Nano Lett. 10, 4206 (2010).

(21) G. Papari, F. Carillo, D. Stornaiuolo, L. Longobardi, F. Beltram, and F. Tafuri, High critical current density and scaling of phase-slip processes in $\mathrm{YBaCuO}$ nanowires. Supercond. Sci. Technol. 25, 035011 (2012).

(22) S. Nawaz, R. Arpaia, F. Lombardi, and T. Bauch, Microwave response of superconducting $\mathrm{YBa} 2 \mathrm{Cu} 3 \mathrm{O} 7-\mathrm{d}$ nanowire bridges sustaining the critical depairing current: Evidence of Josephson-like behavior. Phys. Rev. Lett. 110, 167004 (2013). 
(23) G. P. Papari, A. Glatz, F. Carillo, D. Stornaiuolo, D. Massarotti, V. Rouco, L. Longobardi, F. Beltram, V. M. Vinokur, and F. Tafuri, Geometrical vortex lattice pinning and melting in $\mathrm{YBaCuO}$ submicron bridges. Sci. Rep. 6, 8677 (2016).

(24) R. Cordoba, T. I. Baturina, J. Sesé, A. Y. Mironov, J. M. De Teresa, M. R. Ibarra, D. A. Nasimov, A. K. Gutakovskii, A. V. Latyshev, I. Guillamón, H. Suderow, S. Vieira, M. R. Baklanov, J. J. Palacios, and V. M. Vinokur, Magnetic field-induced dissipation-free state in superconducting nanostructures. Nat. Commun. 4, 1437 (2013).

(25) G. R. Berdiyorov, X. H. Chao, F. M. Peeters, H. B. Wang, V. V. Moshchalkov, and B. Y. Zhu, Magnetoresistance oscillations in superconducting strips: A Ginzburg-Landau study. Phys. Rev. B - Condens. Matter Mater. Phys. 86, 224504 (2012).

(26) Kl H. Kuit, . R. Kirtley, W. van der Veur, C.G. Molenaar, F. J. G.. Roesthuis, A. G. P. Troeman, J. R. Clem, H. Hilgenkamp, H. Rogalla, and J. Flokstra, Vortex trapping and expulsion in thin-film YBCO strips. Phys. Rev. B 2008, 77, 134504.

(27) G. Stan, S. B. Field, and J. M. Martinis, Critical field for complete vortex expulsion from narrow superconducting strips. Phys. Rev. Lett. 2004, 92, 097003.

(28) A. A. Abrikosov, Soviet Physics JETP 19, 988 (1964).

(29) C. P. Bean and J. D. Livingston, Physical Review Letters 12, 14 (1964).

(30) V. V. Shmidt, Soviet Physics JETP 30, 1137 (1970).

(31) M. Tinkham, "Introduction to Superconductivity," in McGraham-Hill International Editors, New York (1996).

(32) S. R. Foltyn, L. Civale, J. L. MacManus-Driscoll, Q. X. Jia, B. Maiorov, H. Wang, and M. Maley, Materials science challenges for high-temperature superconducting wire. Nat. Mater. 6, 631 (2007). 
(33) A. Palau, E. Bartolome, A. Llordes, T. Puig, and X. Obradors, Isotropic and anisotropic pinning in TFA-grown YBa2Cu3O7-x films with BaZrO3 nanoparticles. Supercond. Sci. Technol. 24, 125010 (2011).

(34) A. V. Pan and S. X. Dou, Comparison of small-field behavior in Mg B2, Low- and high-temperature superconductors. Phys. Rev. B - Condens. Matter Mater. Phys. 73, 052506 (2006).

(35) X. Obradors, T. Puig, S. Ricart, M. Coll, J. Gazquez, a Palau, and X. Granados, Growth, nanostructure and vortex pinning in superconducting $\mathrm{YBa} 2 \mathrm{Cu} 3 \mathrm{O} 7$ thin films based on trifluoroacetate solutions. Supercond. Sci. Technol. 2012, 25, 123001.

(36) V. Rouco, D. Massarotti, D. Stornaiuolo, G. P. Papari, X. Obradors, T. Puig, and F. Tafuri, materials Vortex Lattice Instabilities in YBa 2 Cu 3 O 7-x Nanowires. 2018,. 
Graphical TOC Entry

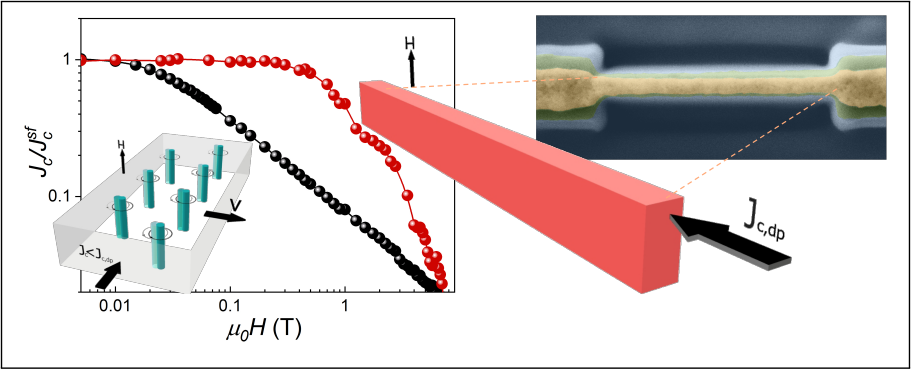

